\title{
A prospective serial MRI study following acute traumatic cervical spinal cord injury
}

\author{
Joost P. H. J. Rutges ${ }^{1,2}$ (1) Brian K. Kwon ${ }^{1,3}$ - Manraj Heran ${ }^{4}$ Tamir Ailon $^{3,5}$ • \\ John T. Street ${ }^{1,3} \cdot$ Marcel F. Dvorak ${ }^{1,3}$
}

Received: 30 June 2016/Revised: 17 March 2017/Accepted: 10 April 2017/Published online: 19 April 2017

(C) The Author(s) 2017. This article is an open access publication

\begin{abstract}
Purpose In acute traumatic cervical spinal cord injury (SCI) patients, we sought to characterize how objective MRI measures of injury change during the first 3 week post-injury.

Methods Six MRI scans each were planned in 19 cervical SCI patients within the first 3 week post-injury. Length of cord edema, maximum spinal cord compression, maximum canal compromise, and presence and length of hematoma were measured.

Results Length of spinal cord edema increased in the first $48 \mathrm{~h}$ after SCI, followed by a gradual decrease in the 3 weeks after injury. This was predominantly seen in the more severe grades of SCI. Hematoma in the spinal cord was seen in all AIS-A and B patients.
\end{abstract}

Marcel F. Dvorak

Marcel.Dvorak@vch.ca

1 Department of Orthopaedics, Blusson Spinal Cord Centre, Vancouver Spine Surgery Institute, University of British Columbia, 818 West 10th Avenue, Vancouver, BC V5Z1M9, Canada

2 Department of Orthopaedics, University Medical Center Utrecht, University of Utrecht, Heidelberglaan 100, 3584 CX Utrecht, The Netherlands

3 International Collaboration on Repair Discoveries (ICORD), Blusson Spinal Cord Centre, University of British Columbia, 818 West 10th Avenue, Vancouver, BC V5Z1M9, Canada

4 Division of Neuroradiology, Department of Radiology, Vancouver General Hospital, University of British Columbia, 899 West 12th Avenue, Vancouver, BC V5Z1M9, Canada

5 Division of Neurosurgery, Department of Surgery, Blusson Spinal Cord Centre, Vancouver Spine Surgery Institute, University of British Columbia, 818 West 10th Avenue, Vancouver, BC V5Z1M9, Canada
Conclusion This study demonstrates the dynamic nature of imaging changes on MRI in the first weeks after injury and highlights the importance of taking into account the timing of imaging when interpreting objective measures of damage.

Keywords Spinal cord injury $\cdot$ MRI $\cdot$ Serial imaging

\section{Introduction}

Acute traumatic spinal cord injury (SCI) is a devastating condition with an annual incidence ranging from 8.0 to 57.8 per million worldwide $[1,2]$. For many years, magnetic resonance imaging (MRI) has routinely been used in the acute setting to characterize the injury and assist in surgical planning. More recently, there has been much interest in utilizing objective measures derived from MRI scans to characterize the severity of neurologic impairment and to predict outcome. The large heterogeneity amongst SCI patients, especially with regard to extent of neurological deficit, injury pattern, overall injury severity, comorbidities, surgical strategies, and rehabilitation care, makes prediction of neurological outcome very difficult [3]. Current methods for predicting outcome based on the initial functional neurologic examination are limited, and there is a great need for objective measures to better classify injury severity and predict outcome.

MRI is currently the gold standard for imaging of SCI and the radiographic characteristics of this injury on MRI are associated with neurological outcome [4]. Since 1988, it has been identified that the presence of spinal cord edema and hematoma portend a worse neurological outcome $[5,6]$. It has been suggested that the extent of spinal cord edema is dynamic in the first days after SCI, based on 
experimental findings [6]. The first indirect clinical evidence was presented in 2008; it was demonstrated that increased time to MRI lead to an increased length in spinal cord edema [7]. Direct evidence that spinal cord edema is dynamic was shown in 2012 when the expansion rate of spinal cord edema between two consecutive MRIs in the first $72 \mathrm{~h}$ after injury was described [8]. However, how spinal cord edema evolves after this initial expansion phase remained unclear.

The work of Aarabi et al. has revealed that to utilize objective MRI features as measures of injury, it is important to acknowledge that the processes captured by MRI are not static; rather, these changes are dynamic and evolve rapidly during the acute period after SCI [8]. When interpreting cord edema on MRI, for example to classify injury severity, or as a surrogate measure of a novel intervention's therapeutic effect, how the extent of cord edema changes over time must be considered. To our knowledge, there are no studies that systematically evaluate the MRI characteristics of traumatic SCI over the first 3 week post-injury. The objective of this study was to characterize the dynamic nature of these MRI characteristics during the first three weeks following SCI using serial MRI scans and to assess the feasibility of conducting such a study in acute SCI patients.

\section{Methods}

\section{Study design}

The study design is prospective descriptive pilot study on MRI characteristics in cervical SCI based on serial scans in patients with cervical SCI.

\section{Patient selection}

Consecutive adult patients (18 years or older) with a traumatic SCI between $\mathrm{C} 1$ and $\mathrm{T} 1$ were eligible for inclusion. Additional inclusion criteria were: admission to the Vancouver General Hospital within $24 \mathrm{~h}$ after the injury; able to communicate in English; and able and willing to provide informed consent. Exclusion criteria were: pathological fractures; SCI due to infection; presence of pacemaker, aneurysm clip, or any other device that is a contraindication to MRI; BMI $>40$ or unable to fit within the MRI scanner; multiple life threatening injuries (ISS $>16$ ) that preclude multiple transports to MRI; any condition that, at the time of admission, prevented complete International Standards for Neurological Classification of Spinal Cord Injury (ISNCSCI) assessment. Based on prior data from our unit, 20 patients were assumed to be sufficient to ensure an adequate patient number in each
American Spinal Injury Association Impairment Scale (AIS) grade in our pilot study.

\section{MRI examinations}

MRI scans were planned at six time points after injurywithin $24 \mathrm{~h}$, within $24-48 \mathrm{~h}, 4$ days $( \pm 6 \mathrm{~h}), 1$ week ( $\pm 12 \mathrm{~h}), 2$ weeks $( \pm 24 \mathrm{~h})$, and 3 weeks $( \pm 48 \mathrm{~h})$. All MRI scans were performed on a $1.5 \mathrm{~T}$ MRI system (Siemens MAGNETUM, Aera, Erlangen, Germany) with a protocol including Sagittal T1, Sagittal T2, Sagittal STIR, and axial T2* GRE imaging sequences.

For Sagittal T2/T1-weighted sequences, images were obtained with a field of view of $220 \mathrm{~mm}$ and an image matrix of $269 \times 384$. The conventional MRI sequences were fast-spin-echo (FSE) T1-weighted sagittal image (TR/ $\mathrm{TE}=533 \mathrm{~ms} / 10 \mathrm{~ms}), \mathrm{T} 2$-weighted sagittal image $(\mathrm{TR} /$ $\mathrm{TE}=3000 \mathrm{~ms} / 84 \mathrm{~ms}$ ), and FSE T2-weighted axial image $(\mathrm{T} 2-\mathrm{WI}) \quad(\mathrm{TR} / \mathrm{TE}=3390 / 98)$ and $\mathrm{T}^{*}$ gradient echo weighted axial image $($ MEDIC) $(\mathrm{TR} / \mathrm{TE}=1030 / 24)$.

\section{Outcome variables}

Patient demographics, injury characteristics, trauma mechanism treatment details, time/date of injury, and all MRI scans were collected in a prospective manner. The ISNCSCI assessment at admission was performed by spine residents and fellows who were specifically trained in this grading instrument.

MRI measurements were performed twice by one observer at different time points at a PACS station. The observer was a fellow in spinal surgery (JR) and certified orthopaedic surgeon with a $\mathrm{PhD}$ in spine research. At the time of the assessments, the observer was blinded for clinical and neurological data. Time interval between the two measurements was at least 1 week. The mean of these two measurements was used as the final outcome measure. MRI outcome variables were: vertical length of cord edema; anatomical point of maximum cord compression; maximum spinal cord compression (MSCC); maximum canal compromise (MCC); and presence and length of hematoma in the spinal cord. Measurements were performed as previously described by Miyanji and Fehlings et al. [9-11]. Briefly, length of spinal cord edema, MSCC, presence and length of hematoma in the spinal cord were determined on the mid-sagittal T2-WI. Spinal cord edema was defined as hyper-intense signal on T2-WI. Hematoma was defined as hypo-intense signal in the spinal cord surrounded by hyper-intense signal on T2-WI. MCC was determined on the initial mid-sagittal T1-WI. On the subsequent scans, we were not able to determine the MCC, since surgical decompression was performed in the majority of the study population. Expansion rate of spinal 
cord edema was calculated by dividing the difference in vertical length of spinal cord edema by the time interval between the injury and the initial MRI or the time interval between consecutive MRIs (Fig. 1).

Reliability of our measurements was assessed by calculating the interclass correlation coefficient (ICC) of vertical length of spinal cord edema, vertical length of hematoma, MSCC, and MCC.

\section{Ethical considerations}

The study protocol was reviewed and approved by the Clinical Research Ethics Board of the University of British Columbia and the Vancouver General Hospital.

\section{Results}

\section{Patient characteristics}

A total of 20 patients were included in this study between February 2015 and April 2016. One patient withdrew after the initial MRI because of claustrophobia, and therefore, MRI scans from 19 patients were available for analysis. The mean age was 57 years, and $74 \%$ were male. Falls were the most common cause of SCI, and fracture dislocation was the most frequent injury morphology. The majority of patients were treated surgically ( $84 \%)$; however, three patients with central cord syndrome without ongoing compression of the spinal cord on the initial MRI were treated conservatively. For all our patients, in this study, mean arterial pressure (MAP) goals were set at $80 \mathrm{mmHg}$ for the first 7 days after injury. None of our patients received dexamethasone as neuroprotective treatment. Neurogenic shock was diagnosed in 5 patients $(26 \%)$. Mean resuscitation volume was $1830 \pm 1900 \mathrm{ml}$. Mean ISS of our patients was $24.3 \pm 8.7$. Mean surgical invasiveness score was $11.6 \pm 5.8$ for the 16 patients who were treated surgically. Mean time interval between injury and surgical stabilisation and decompression was $24.2 \pm 18.3 \mathrm{~h}$ (Table 1 ).

\section{MRI scans}

Seventy-eight percent of the planned MRI scans were performed. The MRI scans most commonly not completed were the scan planned at $48 \mathrm{~h}$ after injury and the scan 3 weeks after injury (42 and 32\%, respectively) (Table 2). The main reason for not obtaining the $48 \mathrm{~h}$ scan was the clinical condition of the patient, with transport of the patient from ICU to the MRI scanner deemed unsafe. The 3 week scans were most commonly missed due to early discharge of the patient. One 0-24 h scan was missed, because the patient was brought directly from the emergency room to the operative theatre in order to facilitate early decompression of the spinal cord. We calculated the ICC to assess the reliability of our MRI measurements. The ICC for vertical length of spinal cord edema, vertical length of hematoma, MSCC, and MCC was $0.872,0.764$, 0.780 , and 0.770 , respectively.

\section{Vertical length of spinal cord edema}

The mean vertical length of spinal cord edema shows a clear pattern in the first 3 weeks after spinal cord injury. There is an increase in mean length of spinal cord edema in the first $48 \mathrm{~h}$ after spinal cord injury, followed by a gradual
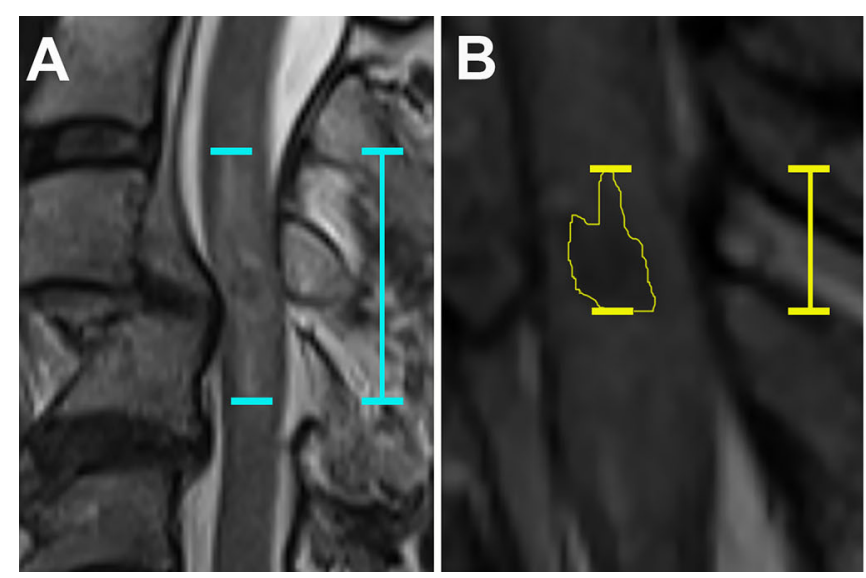

Fig. 1 a Vertical length of cord edema measured as the distance between the most cranial and most distal point of spinal cord edema in the mid-sagittal plane on T2-WI. b Vertical length of hematoma in the spinal cord measured as the distance between the most cranial and most caudal border of the hematoma in the mid-sagittal plane on T2WI. c Maximum spinal cord compromise (MSCC) measured on mid-
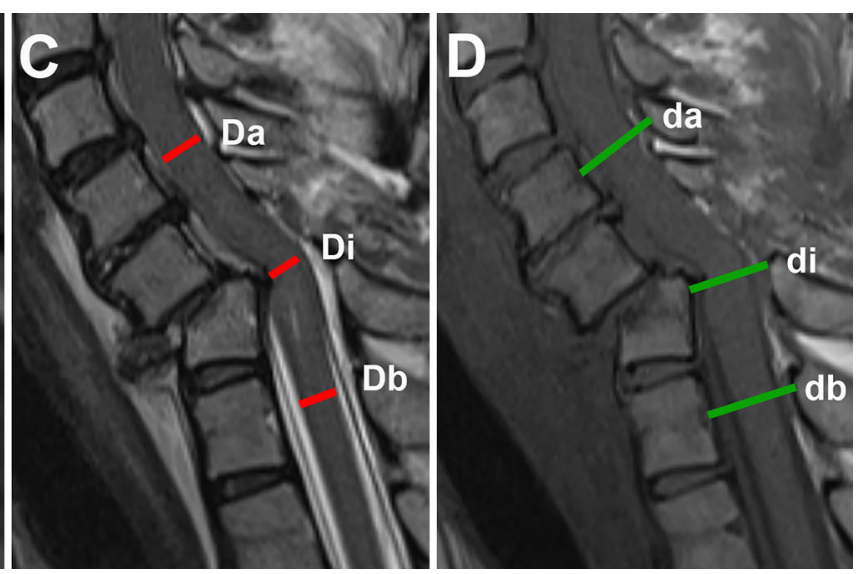

sagittal T2-WI based on spinal cord diameter at the level of injury (Di), first unaffected level above the injury (Da), and first unaffected level below the injury (Db). d Maximum canal compromise (MCC) measured on mid-sagittal T1-WI based on spinal canal diameter at the level of injury (di), first unaffected level above the injury (da), and first unaffected level below the injury $(\mathrm{db})$ 
Table 1 Patients' characteristics

\begin{tabular}{|c|c|}
\hline Number of patients & 19 \\
\hline Female/male (no [\%]) & $5 / 14(26 / 74 \%)$ \\
\hline Age (mean, years [SD]) & $57.2(15.1)$ \\
\hline \multicolumn{2}{|l|}{ Trauma mechanism (no [\%]) } \\
\hline Fall & $11(58 \%)$ \\
\hline Sports & $4(21 \%)$ \\
\hline MVA & $3(16 \%)$ \\
\hline Other & $1(5 \%)$ \\
\hline \multicolumn{2}{|l|}{ Injury morphology (no [\%]) } \\
\hline Fracture dislocation & $10(53 \%)$ \\
\hline Central cord syndrome & $5(26 \%)$ \\
\hline Hyper extension injury & $4(21 \%)$ \\
\hline \multicolumn{2}{|l|}{ AIS grade at admission (no [\%]) } \\
\hline AIS-A & $8(42 \%)$ \\
\hline AIS-B & $2(11 \%)$ \\
\hline AIS-C & $4(21 \%)$ \\
\hline AIS-D & $5(26 \%)$ \\
\hline \multicolumn{2}{|c|}{ Neurological level of injury (no [\%]) } \\
\hline $\mathrm{C} 2$ & $2(11 \%)$ \\
\hline $\mathrm{C} 3$ & $1(5 \%)$ \\
\hline $\mathrm{C} 4$ & $7(37 \%)$ \\
\hline C5 & $6(32 \%)$ \\
\hline C6 & $2(11 \%)$ \\
\hline $\mathrm{T} 1$ & $1(5 \%)$ \\
\hline \multicolumn{2}{|l|}{ Treatment (no [\%]) } \\
\hline Surgical anterior approach & $7(37 \%)$ \\
\hline Surgical posterior approach & $5(26 \%)$ \\
\hline Surgical circumferential $/ 360^{\circ}$ & $4(21 \%)$ \\
\hline Conservative management & $3(16 \%)$ \\
\hline
\end{tabular}

$S D$ standard deviation, $M V A$ motor vehicle accident, AIS American Spinal Injury Association Impairment Scale

Table 2 Percentage of completed planned MRI scans per time point

\begin{tabular}{ll}
\hline Time point & $\begin{array}{l}\text { Percentage of } \\
\text { completed scans }(\%)\end{array}$ \\
\hline $0-24 \mathrm{~h}$ & 95 \\
$48 \mathrm{~h}$ & 58 \\
$96 \mathrm{~h}$ & 79 \\
1 week & 89 \\
2 week & 79 \\
3 week & 68 \\
\hline
\end{tabular}

decrease in the following 3 weeks (Fig. 2a). The change in expansion rate of spinal cord edema is in line with this pattern (Fig. 3a). The stratified data for AIS grade demonstrate that this pattern was mainly present in the AIS-A patients. Length of spinal cord edema in the AIS-B,
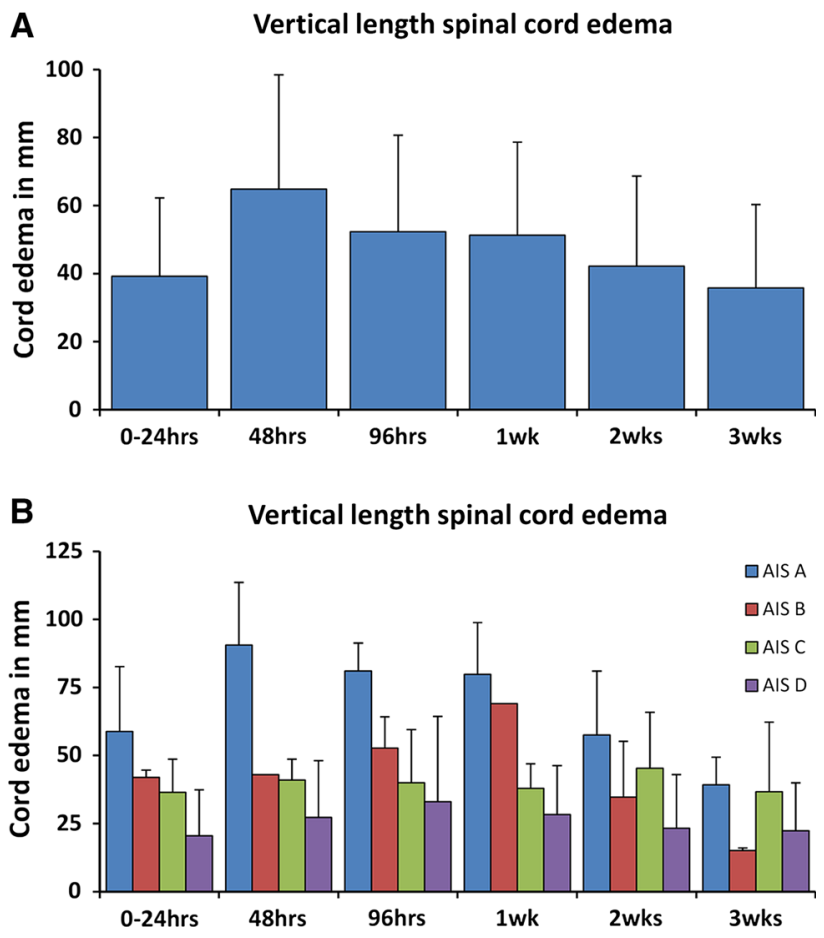

Fig. 2 Mean length of spinal cord edema for each time point (a). Mean length of spinal cord edema for each time point stratified for AIS grade per time point (b). Error bars indicate SD
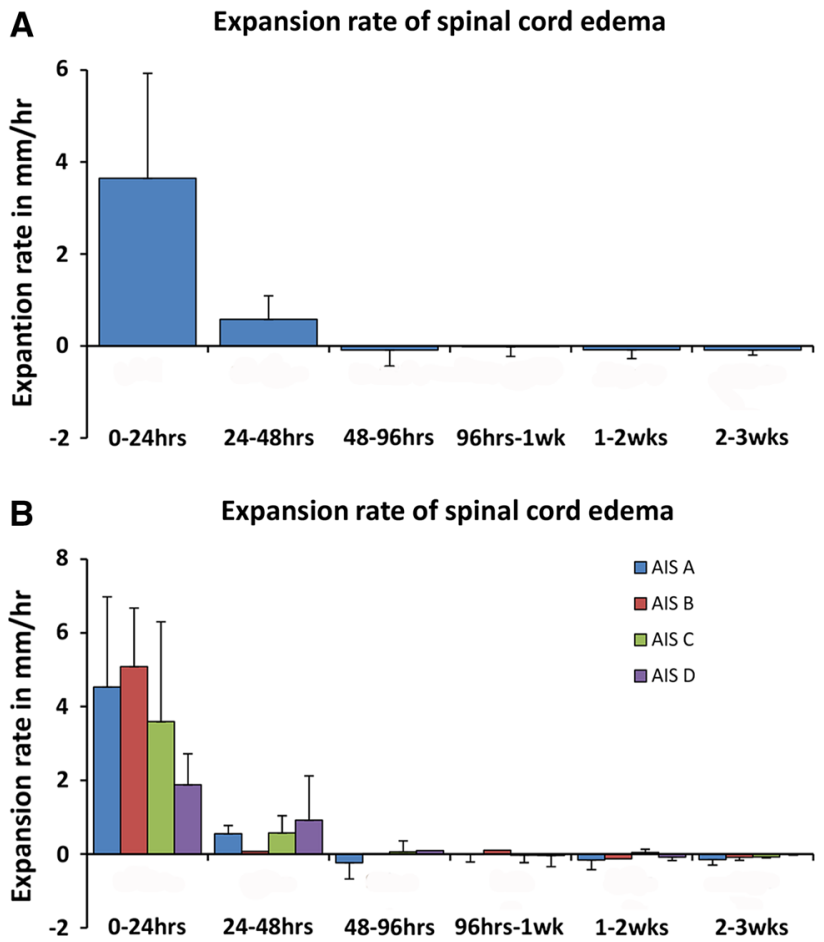

Fig. 3 Mean expansion rate of spinal cord edema for each time point (a). Mean expansion rate of spinal cord edema each time point stratified for AIS grade (b). A negative value represents a decrease in spinal cord edema. Error bars indicate SD 
-C, and -D patients was much more stable over time (Fig. 2b). The expansion rate for spinal cord edema stratified according to AIS score further supports this finding by showing the highest expansion rates in the AIS-A patients (Fig. 3b). A clinical example of the temporal change in length of spinal cord edema is shown in Fig. 4.

\section{Vertical length of spinal cord hematoma}

Hematoma in the spinal cord was found in all AIS-A and B patients, and in $50 \%$ of the AIS-C patients (Table 3). If a hematoma was seen, it was usually identified on the initial MRI and remained present until the final MRI at 3 weeks after injury. However, in one AIS-A and one AIS-C patient, the hematoma was not present at the initial MRI and was identified at the second scan. In one AIS-B patient, only a small hematoma was seen on the 48-h MRI scan and not on any of the other scans. No hematomas were seen in the AIS-D patients (Table 3).

The mean length of the hematoma in the spinal cord increased in the first $48 \mathrm{~h}$ after injury, and then gradually decreased in the 3 weeks after injury (Fig. 5). A clinical example of the change in length of the hematoma in the first 3 weeks after SCI is shown in Fig. 4.

\section{Spinal cord compression and spinal canal compromise}

Mean MSCC on the initial MRI ranged from 14 to $19 \%$ (Fig. 6a). Mean MCC on the initial MRI ranged from 15 to 26\% (Fig. 6b). Minimally higher MSCC levels were found in the more severe SCIs, whereas no relation was identified between MCC and AIS grade (Fig. 6a, b).

The MSCC decreased after the initial MRI as a result of surgical decompression. The negative MSCC values indicate swelling of the spinal cord (Fig. 7a). Spinal cord swelling was predominantly seen in the more severe SCI (Fig. 7b).

\section{Discussion}

This prospective study, for the first time, describes the temporal changes of MRI characteristics in SCI during the first 3 weeks after injury. We identified an increase in vertical length of spinal cord edema in the first $48 \mathrm{~h}$ after SCI, followed by a gradual decrease in the 3 weeks after injury. Not surprisingly, the greatest lengths of spinal cord edema and hematoma were found in those with the most
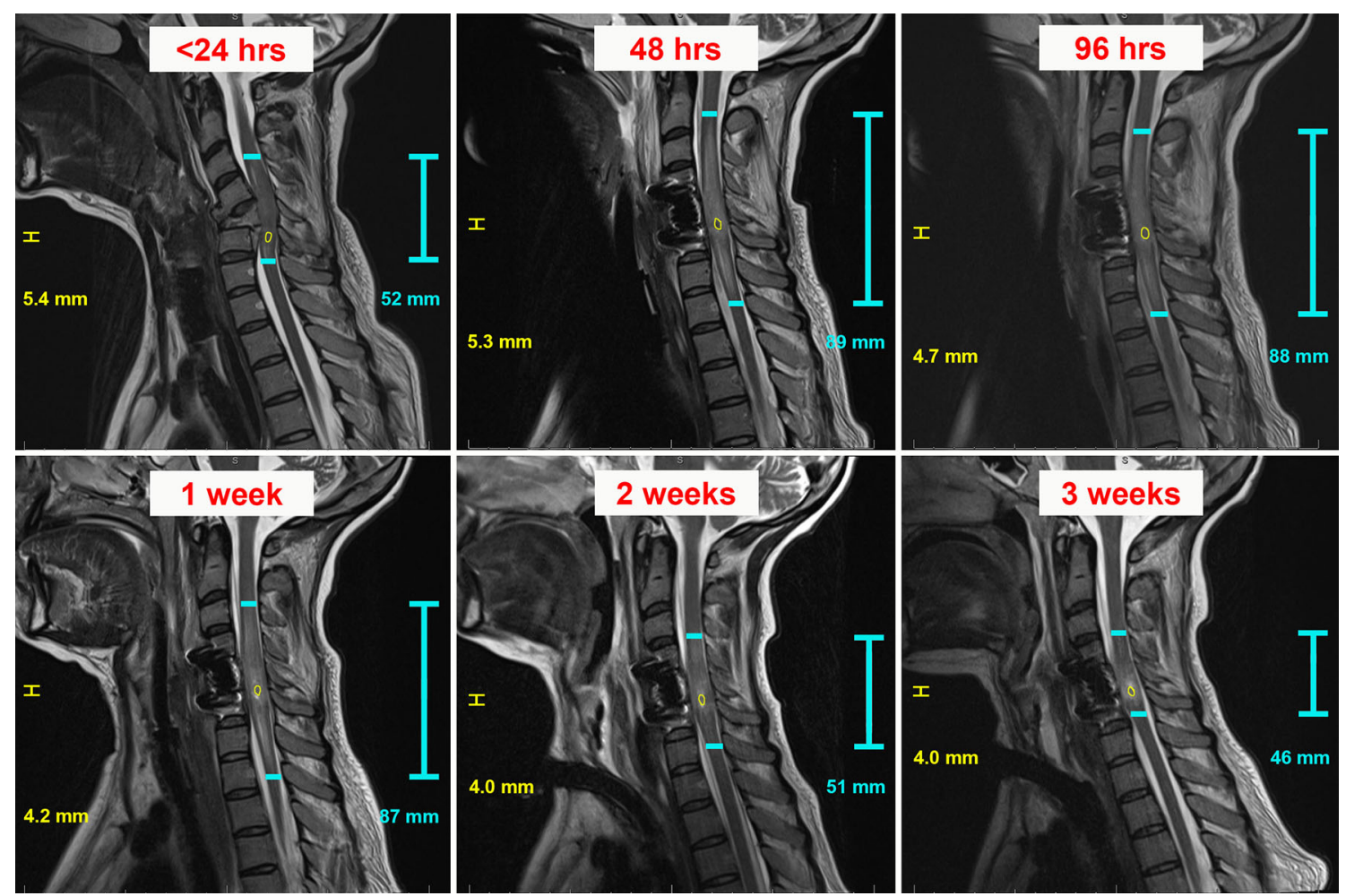

Fig. 4 Clinical example of changes in MRI characteristics for each time point in a patient with a C5-6 fracture dislocation, C5 AIS-A, treated with a $\mathrm{C} 5$ vertebrectomy, cage, and anterior plate fixation. The short horizontal blue lines indicate the cranial and caudal extent of the spinal cord edema, whereas the vertical blue lines indicate the length of spinal cord edema as indicated in $\mathrm{mm}$. The yellow line drawn around the borders of the hematoma in the spinal cord to demonstrate the size of the hematoma at each time point, whereas the vertical yellow lines indicate the length of the hematoma as indicated in $\mathrm{mm}$ 
Table 3 Presence of hematoma in the spinal cord

\begin{tabular}{lclc}
\hline AIS grade at admission & Hematoma $(\%)$ & Mean length of hematoma (mm [SD]) & No hematoma (\%) \\
\hline AIS-A & 100 & $7.6(4.7)$ & 0 \\
AIS-B & 100 & $4.7(3.1)$ & 0 \\
AIS-C & 50 & $1.9(0.9)$ & 50 \\
AIS-D & 0 & - & 100 \\
\hline
\end{tabular}

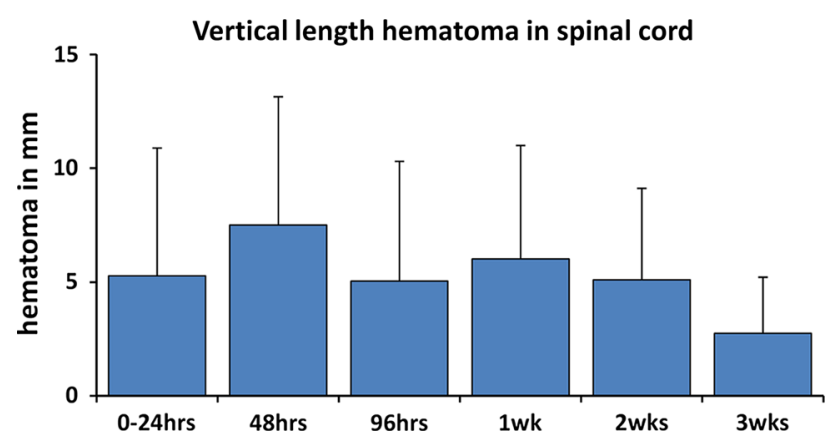

Fig. 5 Mean length of hematoma in the spinal cord for each time point. Error bars indicate SD
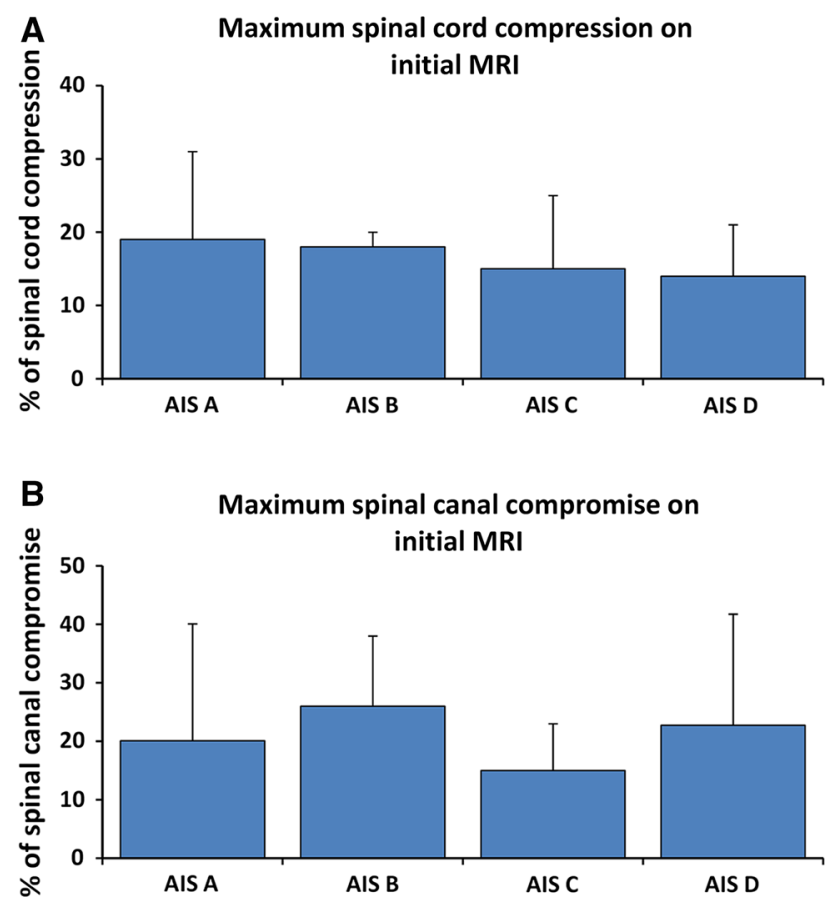

Fig. 6 Mean maximum spinal cord compression (MSCC) on the initial MRI for each AIS grade (a) Mean maximum canal compromise (MCC) on the initial MRI for each AIS grade (b). Error bars indicate SD

severe (AIS-A and B) injuries. Hematoma in the spinal cord was seen in all AIS-A and B patients, in 50\% of the AIS-C patients, and in none of the AIS-D patients.

The first serial MRI study in SCI was performed by Shimada et al. and was published in 1999 [12]. This study reports MRI characteristics of SCI in the first year after injury and showed a decrease in length of spinal cord
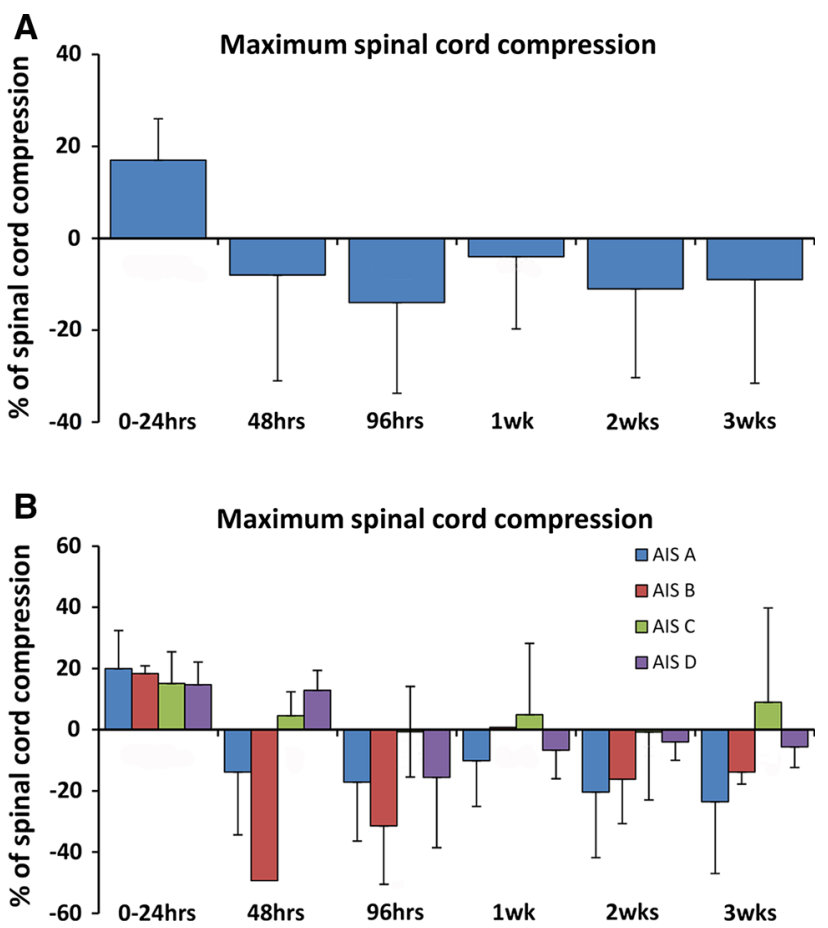

Fig. 7 Mean maximum spinal cord compression (MSCC) for each time point (a). Mean MSCC for each time point stratified for AIS grade (b). A negative value represents spinal cord swelling. Error bars indicate SD

edema between the initial MRI and the scan performed 3 weeks after the injury [12]. Since no serial MRI scans were performed in the first week after SCI, this study was not able to assess for any increase in spinal cord edema during the first $48 \mathrm{~h}$ after injury [12].

The first direct evidence that spinal cord edema increases in the first days after spinal cord injury was provided by Aarabi et al. in [8]. Two consecutive scans in the first days after SCI were performed in 42 patients and showed an increase in spinal cord edema during the time interval between the scans [8]. The described spinal edema expansion rate of $0.9 \mathrm{~mm} / \mathrm{h}$ by Aarabi et al. is comparable to the mean $0.6 \mathrm{~mm} / \mathrm{h}$ that we found in our $24-48 \mathrm{~h}$ interval [8]. As shown in the current study, the expansion rate steadily decreases during the first $48 \mathrm{~h}$ after SCI. Therefore, the lower value in the current study could be explained by the timing of the initial MRI. In Aarabi's study, the initial MRI was performed a mean of $6.8 \mathrm{~h}$ after injury, whereas in the current study, this was $14.6 \mathrm{~h}$ [8]. 
Hematoma in the spinal cord has been associated with a worse neurological prognosis and is one of the most important predictors for neurological outcome [5, 13]. Spinal cord hematoma in SCI is predominantly seen in AIS-A and B patients, with reported ranges being 50-100\% and 14-72\%, respectively [9, 14-16]. However, hemorrhage in AIS-C patients and in combined patient cohorts of incomplete injuries (AIS-B-D) has also been reported $[9,14,16]$. These findings are all based on single MRI studies and in a heterogeneous time window after injury, ranging from $<24$ h to 12 days. This might explain why the percentage of spinal cord hematomas in the AIS-B and C patients in the current study was higher than reported by the previous studies, especially since $25 \%$ of the hematomas in this study were not identified on the initial MRI.

Length of hematoma is sparsely reported in literature. Boldin et al. report a mean length of $10.5 \mathrm{~mm}$ in AIS-A patients and a mean of $4.0 \mathrm{~mm}$ in incomplete injuries [14]. These findings are comparable with the hematoma length found in the current study.

MCC and MSCC as methods to quantify spinal canal compromise and spinal cord compression/swelling after traumatic SCI were introduced by Fehlings and Furlan et al. $[11,17]$. Several studies have used these methods to analyse canal compromise and cord compression after traumatic SCI $[9,13,16,18]$. MCC and MSCC reported in these studies range between $22-62 \%$ and $23-58 \%$, respectively, for complete injuries and $14-38 \%$ and 20-52\%, respectively, for incomplete injuries [9, 13, 16]. The MCC and MSCC reported in the current study fall within the lower percentile reported in literature. When compared to other studies, the current study consists of a relatively large number of injuries caused by low energy mechanisms (falls) that might result in a lower mean MCC and MSCC [9, 13, 16]. Recently, the level of pre-existing spinal cord compression in patients with SCI without bone injury, as measured by the MSCC, has been related to the severity of neurological injury [19]. In the current study, only 5 patients without bone injury were included, and therefore, no relation between MSCC and AIS grade could be identified; nevertheless, all these patients had pre-existing cord compromise with a mean MSCC of $16 \%$.

The observed swelling of the spinal cord on MRI is a well-known qualitative variable in SCI and has been associated with more severe injuries and worse neurological prognosis $[9,18,20]$. The spinal cord swelling found in the current study was predominantly seen in the AIS-A and $B$ patients and, therefore, in line with our current understanding.

A secondary aim of the current study was to evaluate if it was feasible to conduct a serial MRI study in the acute phase after SCI. In total, $78 \%$ of the planned MRIs were actually performed. The most commonly missed MRI was the scan that was planned at $48 \mathrm{~h}$ after injury. During the 24-48 h interval, the patients in our study were often undergoing surgery or just recovering from it, often in the ICU, intubated, sedated, and on vasopressors. Therefore, in some cases, transfer to the MRI scanner was deemed too risky for a research MRI. In addition, the MRI at 3 weeks after the injury was often missed, especially for the AIS-C and $\mathrm{D}$ patients. These patients were usually already discharged to a rehabilitation facility without resources to accommodate the transport to our hospital for the followup MRI.

There are very few serial MRI studies performed during the first weeks after a trauma or other acute event. One serial MRI study conducted in the first weeks after a stroke achieved a total of $88 \%$ completed scans [21]. Based on the fact that the patient population in the current study often required acute surgery, prolonged intubation, and ventilation, the $78 \%$ completed scans, in our opinion, should be considered as a reasonable achievement.

Clearly, missing a substantial part of our patients' data is a limitation of this study. However, this might be an inevitable consequence of studying severely injured patients during the acute phase after their trauma. Another limitation of our study is the small sample size per AIS grade, especially the AIS-B group $(n=2)$. Nevertheless, the other incomplete (AIS-C and D) and the complete injuries (AIS-A) are well represented, and therefore, it is still reasonable to draw conclusions from this descriptive pilot study.

This study was designed as a descriptive pilot study with a small number of patients and this limits the statistical analysis that can be performed. This study was specifically designed to identify the change of MRI characteristics over time enabling us to design further studies with an appropriate sample size. For this study, a single observer with high ICC values is considered adequate to identify the dynamic nature of MRI characteristics in SCI; nevertheless, for future studies, multiple observers will be used.

The relation between the dynamics of MRI characteristics and neurological recovery is an important subject of future serial studies. Furthermore, it will be important to identify which time point for an MRI scan after SCI has the best prognostic value for neurological recovery. The recently introduced BASIC classification for SCI strongly correlates with potential neurological recovery [22]. Including the BASIC classification in future serial MRI studies is an important step to determine the time point at which an MRI has the best prognostic value [22]. 


\section{Conclusions}

This study demonstrates a clear pattern of temporal changes in the MRI characteristics that accompany the first 3 weeks after SCI on serial images. We have shown that serial MRI studies in traumatic SCIs are feasible; however, it should be anticipated that some of the planned scans may not be performed due to the clinical condition of the patient. Based on the current study, it is important to realise that spinal cord edema on MRI is dynamic. This should be taken into account when interpreting SCI on MRI for clinical or scientific purposes. This study provides further support for the current understanding that many morphologic changes occur in the injured spinal cord in the first 48-72 h after injury. Assuming that these morphologic changes are manifestations of the pathophysiologic processes that occur post-injury, the findings would support the notion that significant secondary damage is occurring in these first few days and it is a time period, well suited for neuroprotective agents. Importantly, for novel neuroprotective interventions being tested in acute SCI, the alteration in these measurable dynamic changes on MRI may prove to be a useful surrogate outcome measure of biological effect. Such biomarkers would be highly useful in the testing of novel therapies, but in the case of early MRI measures, there is definitely the need to understand the dynamic nature of the changes.

Acknowledgements We would like to acknowledge Allan Aludino, Leilani Reichl, Angela Tsang, Leanna Ritchie, Lise Belanger, and Jiwan Gill from the Vancouver Spine Research Program for their help with study. We are grateful for the assistance from individuals within many departments within Vancouver General Hospital including Dave Ferguson MD and Donna Sheppard from Radiology, Suzie Logie and all the respiratory therapists from Respiratory Therapy, Jackson Lam, and Denise Foster from ICU, Brendan Tompkins, and all the registered nurses from the Acute Spine Unit, and Mary Heim from Patient Transport.

\section{Compliance with ethical standards}

Conflict of interest Authors MD and BK have received research funding from the Rick Hansen Institute. Author MD receives consulting and royalties from Medtronic which is not related to the subject of this study. Authors MD, BC, TA, and JS receive academic funding for fellowship support from AO Spine International, Medtronic, and Synthes.

Funding This study was funded by the Rick Hansen Institute and The Paetzold Chair in SCI.

Ethical considerations The study protocol was reviewed and approved by the Clinical Research Ethics Board of the University of British Colombia, Vancouver, British Colombia, Canada, study ID H14-01848.

Open Access This article is distributed under the terms of the Creative Commons Attribution 4.0 International License (http://crea tivecommons.org/licenses/by/4.0/), which permits unrestricted use, distribution, and reproduction in any medium, provided you give appropriate credit to the original author(s) and the source, provide a link to the Creative Commons license, and indicate if changes were made.

\section{References}

1. van den Berg ME, Castellote JM, Mahillo-Fernandez I, de PedroCuesta J (2010) Incidence of spinal cord injury worldwide: a systematic review. Neuroepidemiology 34(3):184-192. doi:10. $1159 / 000279335$

2. Singh A, Tetreault L, Kalsi-Ryan S, Nouri A, Fehlings MG (2014) Global prevalence and incidence of traumatic spinal cord injury. Clin Epidemiol 6:309-331. doi:10.2147/CLEP.S68889

3. Krishna V, Andrews H, Varma A, Mintzer J, Kindy MS, Guest J (2014) Spinal cord injury: how can we improve the classification and quantification of its severity and prognosis? J Neurotrauma 31(3):215-227. doi:10.1089/neu.2013.2982

4. Bozzo A, Marcoux J, Radhakrishna M, Pelletier J, Goulet B (2011) The role of magnetic resonance imaging in the management of acute spinal cord injury. J Neurotrauma 28(8):1401-1411. doi:10.1089/neu.2009.1236

5. Kulkarni MV, Bondurant FJ, Rose SL, Narayana PA (1988) 1.5 tesla magnetic resonance imaging of acute spinal trauma. Radiographics 8(6):1059-1082. doi:10.1148/radiographics.8.6. 3205929

6. Bondurant FJ, Cotler HB, Kulkarni MV, McArdle CB, Harris Jr JH (1990) Acute spinal cord injury: a study using physical examination and magnetic resonance imaging. Spine 15(3):161-168 (Phila Pa 1976)

7. Leypold BG, Flanders AE, Burns AS (2008) The early evolution of spinal cord lesions on MR imaging following traumatic spinal cord injury. AJNR Am J Neuroradiol 29(5):1012-1016. doi:10. 3174/ajnr.A0962

8. Aarabi B, Simard JM, Kufera JA, Alexander M, Zacherl KM, Mirvis SE, Shanmuganathan K, Schwartzbauer G, Maulucci CM, Slavin J, Ali K, Massetti J, Eisenberg HM (2012) Intramedullary lesion expansion on magnetic resonance imaging in patients with motor complete cervical spinal cord injury. J Neurosurg Spine 17(3):243-250. doi:10.3171/2012.6.SPINE12122

9. Miyanji F, Furlan JC, Aarabi B, Arnold PM, Fehlings MG (2007) Acute cervical traumatic spinal cord injury: MR imaging findings correlated with neurologic outcome-prospective study with 100 consecutive patients. Radiology 243(3):820-827. doi:10.1148/ radiol. 2433060583

10. Fehlings MG, Rao SC, Tator CH, Skaf G, Arnold P, Benzel E, Dickman C, Cuddy B, Green B, Hitchon P, Northrup B, Sonntag V, Wagner F, Wilberger J (1999) The optimal radiologic method for assessing spinal canal compromise and cord compression in patients with cervical spinal cord injury. Part II: Results of a multicenter study. Spine 24(6):605-613 (Phila Pa 1976)

11. Fehlings MG, Furlan JC, Massicotte EM, Arnold P, Aarabi B, Harrop J, Anderson DG, Bono CM, Dvorak M, Fisher C, France J, Hedlund R, Madrazo I, Nockels R, Rampersaud R, Rechtine G, Vaccaro AR (2006) Interobserver and intraobserver reliability of maximum canal compromise and spinal cord compression for evaluation of acute traumatic cervical spinal cord injury. Spine 31(15):1719-1725. doi:10.1097/01.brs.0000224164.43912.e6 (Phila Pa 1976)

12. Shimada K, Tokioka T (1999) Sequential MR studies of cervical cord injury: correlation with neurological damage and clinical outcome. Spinal Cord 37(6):410-415

13. Gupta R, Mittal P, Sandhu P, Saggar K, Gupta K (2014) Correlation of qualitative and quantitative MRI parameters with 
neurological status: a prospective study on patients with spinal trauma. J Clin Diagn Res RC8(RC11):13-17. doi:10.7860/JCDR/ 2014/9471.5181

14. Boldin C, Raith J, Fankhauser F, Haunschmid C, Schwantzer G, Schweighofer F (2006) Predicting neurologic recovery in cervical spinal cord injury with postoperative MR imaging. Spine 31(5):554-559. doi:10.1097/01.brs.0000201274.59427.a4 (Phila Pa 1976)

15. Schaefer DM, Flanders A, Northrup BE, Doan HT, Osterholm JL (1989) Magnetic resonance imaging of acute cervical spine trauma: correlation with severity of neurologic injury. Spine 14(10):1090-1095 (Phila Pa 1976)

16. Song KJ, Ko JH, Choi BW (2016) Relationship between magnetic resonance imaging findings and spinal cord injury in extension injury of the cervical spine. Eur J Orthop Surg Traumatol 26(3):263-269. doi:10.1007/s00590-015-1731-1

17. Furlan JC, Fehlings MG, Massicotte EM, Aarabi B, Vaccaro AR, Bono CM, Madrazo I, Villanueva C, Grauer JN, Mikulis D (2007) A quantitative and reproducible method to assess cord compression and canal stenosis after cervical spine trauma: a study of interrater and intrarater reliability. Spine 32(19):2083-2091. doi:10.1097/BRS.0b013e318145a91c (Phila Pa 1976)
18. Singh R, Kumar RR, Setia N, Magu S (2015) A prospective study of neurological outcome in relation to findings of imaging modalities in acute spinal cord injury. Asian $\mathbf{J}$ Neurosurg 10(3):181-189. doi:10.4103/1793-5482.161166

19. Oichi T, Oshima Y, Okazaki R, Azuma S (2016) Preexisting severe cervical spinal cord compression is a significant risk factor for severe paralysis development in patients with traumatic cervical spinal cord injury without bone injury: a retrospective cohort study. Eur Spine J 25(1):96-102

20. Kalfas I, Wilberger J, Goldberg A, Prostko ER (1988) Magnetic resonance imaging in acute spinal cord trauma. Neurosurgery 23(3):295-299

21. Schwamm LH, Koroshetz WJ, Sorensen AG, Wang B, Copen WA, Budzik R, Rordorf G, Buonanno FS, Schaefer PW, Gonzalez RG (1998) Time course of lesion development in patients with acute stroke: serial diffusion- and hemodynamic-weighted magnetic resonance imaging. Stroke 29(11):2268-2276

22. Talbott JF, Whetstone WD, Readdy WJ, Ferguson AR, Bresnahan JC, Saigal R, Hawryluk GW, Beattie MS, Mabray MC, Pan JZ, Manley GT, Dhall SS (2015) The Brain and Spinal Injury Center Score: a novel, simple, and reproducible method for assessing the severity of acute cervical spinal cord injury with axial T2-weighted MRI findings. J Neurosurg Spine 23(4):495-504 